\title{
Isthmische Spondylolyse und Spondylolisthese
}

\author{
Frank Kandziora, Matti Scholz, Andreas Pingel, Reinhard Hoffmann
}

\section{Zusammenfassung}

Die Spondylolyse und Spondylolisthese ist ein komplexes Krankheitsbild, das häufig bereits im Kindes- und Jugendalter relevant wird. Nur die symptomatischen Patienten bedürfen der detaillierten Diagnostik und der Therapie. Beim Vorliegen von Risikofaktoren muss eine mögliche Progression der Spondylolisthese regelmäßig überprüft werden. Neben der konservativen Therapie stehen bis zum ca. 25. Lebensjahr rekonstruktive Eingriffe im Bereich der Pars interarticularis zur Verfügung. Fusionsoperation bei Spondylolisthesen können mit oder ohne Reposition (in situ fusion) durchgeführt werden. Obwohl speziell die operative Therapie der hochgradigen Spondylolisthese komplikationsträchtig ist, sind bei rechtzeitiger und adäquater Behandlung regelhaft gute und sehr gute Ergebnisse zu erzielen.

\section{Isthmic Spondylolysis and Spondylolisthesis}

Spondylolysis and spondylolisthesis are complex disease patterns that often become relevant already in childhood and adolescence. Only symptomatic patients need detailed diagnosis and therapy. If risk factors are evident, progression of spondylolisthesis has to be re-evaluated at regular intervals. In addition to conservative treatment reconstruction of the pars interarticularis is possible until patients reach the age of 25 . Fusion procedures can be performed with or without reduction (in situ fusion). Although surgery includes several potential complications, especially in high-grade spondylolisthesis, an early and adequate treatment regularly yields good and very good results.

\section{Definition und Ätiologie}

Unter Spondylolyse versteht man eine uni- oder bilaterale Spaltbildung in der Pars interarticularis des Wirbelbogens. Bei der Spondylolisthese handelt es sich um eine Ventralverlagerung des kranialen Wirbelkörpers in Relation zum kaudalen Wirbelkörper.

Obwohl die Erstbeschreibung der Spondylolisthese bereits im Jahr 1782 durch Herbinaux erfolgte, ist die Genese der Spondylolyse und Spondylolisthese nach wie vor ungenügend geklärt. Allgemein anerkannt ist, dass es sich dabei um ein multifaktorielles Geschehen handelt, auf der Basis mechanischer, genetischer und hormoneller Faktoren.

OP-JOURNAL 2009; 25: 106-111

(c) Georg Thieme Verlag KG Stuttgart · New York DOI $10.1055 / \mathrm{s}-0029-1186062$

\section{Epidemiologie}

Nach Logroscino [8] sind im 6. Lebensjahr ca. 4\% aller Knaben von einer Spondylolyse betroffen. Im Erwachsenenalter findet sich eine Spondylolyse bei ca. 6\% der Männer, bei Frauen ist sie nur halb so häufig. Hinsichtlich der Inzidenz und Prävalenz bestehen hohe regionale und ethnische Unterschiede. So lässt sich bei schwarzen Frauen eine Spondylolyserate von nur ca. $1 \%$ nachweisen, wohingegen bei Eskimos 54\% der Bevölkerung eine Spondylolyse aufweisen. Am häufigsten findet sich die Spondylolyse im Bewegungssegment L5/S1 (82\%), gefolgt vom Bewegungssegment L4/5 (11\%).

\section{Klassifikation}

Die Spondylolisthese wird am häufigsten nach Wiltse [16] eingeteilt, dabei werden 6 Typen unterschieden (Tab. 1).
Der häufigste Typ ist der Typ III, der im Rahmen von degenerativen Veränderungen der Lendenwirbelsäule auftritt.

Als „klassische Spondylolyse und Spondylolisthese" wird jedoch der Typ II, die isthmische Spondylolyse, verstanden (Abb. 1-5). In dieser Gruppe existieren 3 Unterformen: Die 1. und häufigste Untergruppe beschreibt die einfache Spondylolyse der Pars interarticularis (Abb. 1-5). In der 2. Untergruppe kommt es zur sekundären Elongation der Pars interarticularis als Folge einer Lyse. Schließlich wird noch eine akute Fraktur der Pars interarticularis unterschieden. Dieser zuletzt genannte Untertyp grenzt sich vom Typ IV dadurch ab, dass die traumatische Spondylolyse in einer anderen Region als der Pars interarticularis nachzuweisen ist.

Von zunehmender Bedeutung ist auch die Marchetti-Bartolozzi-Klassifikation [9], die angeborene (hoch dysplastisch und gering dysplastische Formen) sowie

Tab. 1 Einteilung der Spondylolisthese nach Wiltse [16].

\begin{tabular}{l} 
Typ I: angeboren oder dysplastisch \\
\hline - axiale Ausrichtung der Gelenkfortsätze \\
\hline $\begin{array}{l}\text { - sagittale Ausrichtung der Gelenkfort- } \\
\text { sätze }\end{array}$ \\
\hline - andere kongenitale Anomalien \\
\hline Typ II: isthmisch \\
\hline - Lyse in der Pars interarticularis \\
\hline - sekundäre Elongation der Pars interarti- \\
cularis \\
\hline - akute Pars-interarticularis-Fraktur \\
\hline Typ III: degenerativ \\
\hline $\begin{array}{l}\text { Typ IV: traumatisch (andere Lokalisation } \\
\text { als Pars interarticularis) }\end{array}$ \\
\hline Typ V: pathologisch \\
\hline Typ VI: postoperativ
\end{tabular}




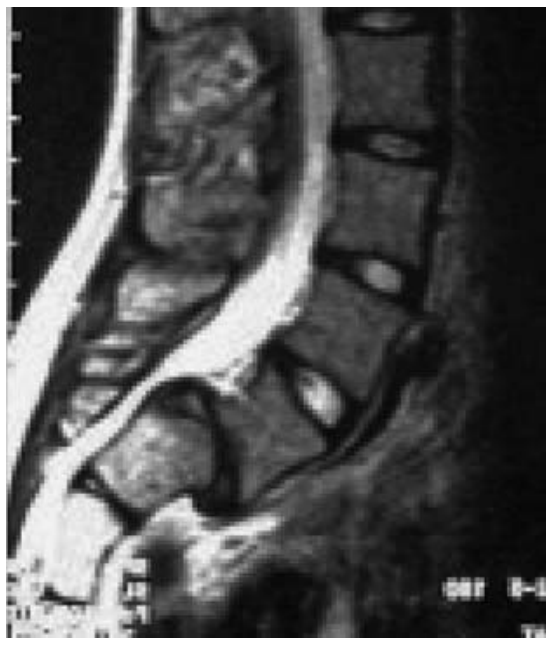

Abb. 1 Sagittales MRT (T2) eines 8-jährigen Mädchens. Isthmische Spondylolisthese (Meyerding Grad I) bei Spondylolyse mit domförmigem Sakrum und trapezförmigem 5. Lendenwirbelkörper.

erworbene Formen der Spondylolyse unterscheidet.

\section{Klinische Symptomatik}

Die Beschwerdesymptomatik bei Spondylolyse und Spondylolisthese ist durch 3 Problemkreise definiert.

\section{Lumbalgie}

Die Lumbalgie ist meist belastungsabhängig, sie kann isthmischen, diskogenen oder muskulären Ursprungs sein. Vor allen Dingen die isthmischen Beschwerden, d.h. im Bereich der Spondylolyse selbst generierte Beschwerden, sind bei Kindern und Jugendlichen häufig anzutreffen.

\section{Ischialgie}

Eine neurologische Ausfallsymptomatik ist meist bei fortgeschrittener Spondylolyse mit Spondylolisthese anzutreffen. Das Wirbelgleiten kann dabei zu einer zentralen Spinalstenose führen, die eine Cauda-equina-Symptomatik mit Blasenund Mastdarmschwäche sowie Hüftlendenstrecksteife durch Spasmus der ischiokoralen Muskulatur zur Folge haben kann. Die Ischialgie, die regelhaft die Nervenwurzel L5 und seltener auch die Nervenwurzel S1 betrifft, kann durch eine subpedikuläre Einengung durch fibrokartilaginäres Gewebe im Parsbereich, durch eine foraminale Stenose bei neuroforaminaler Kompression oder in der Folge eines „Far-out-Syndroms“ durch Kompression des Nervs im Bereich des
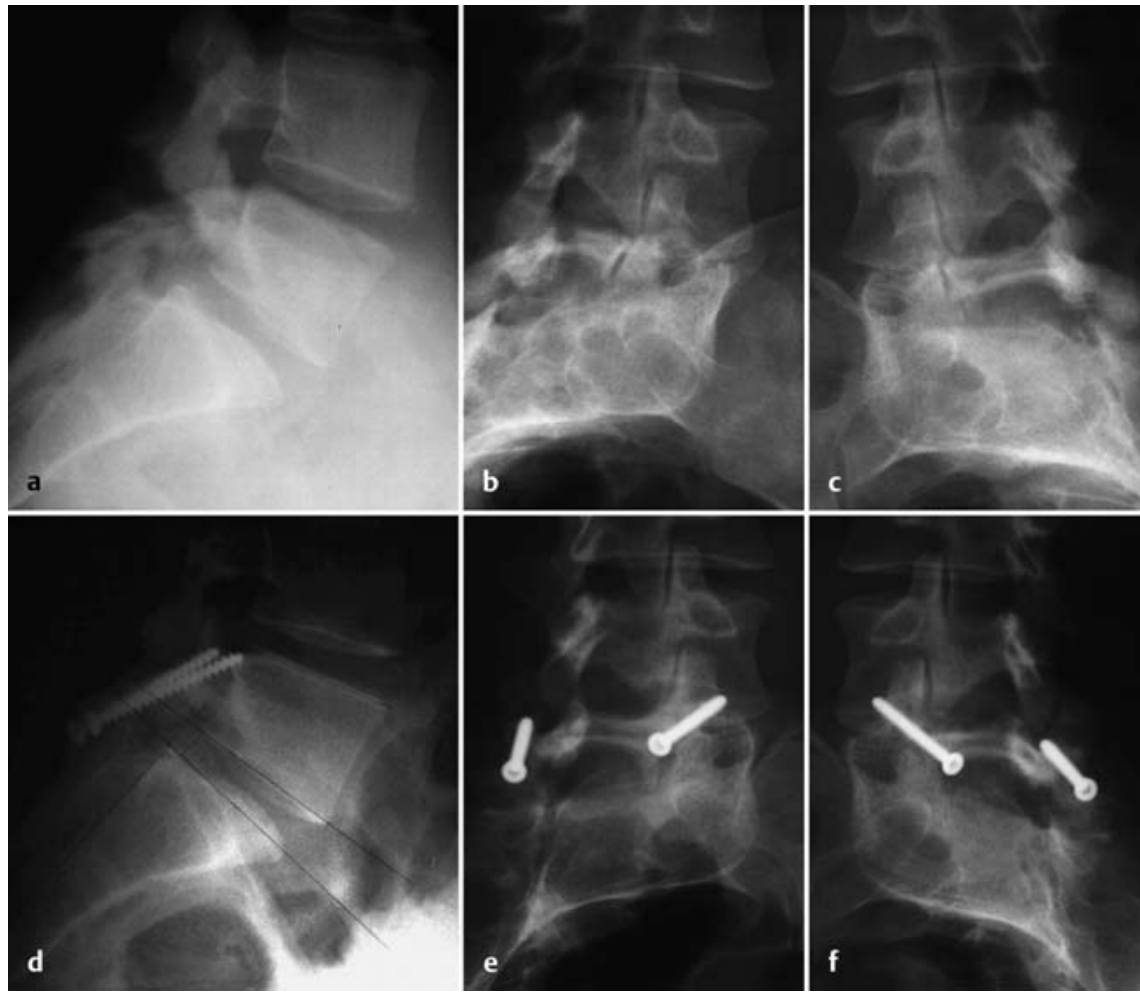

Abb. 2 a bis f 15-jähriger Knabe mit symptomatischer Spondylolyse: (a) Röntgenologische Darstellung des Hundehalsbands auf den Schrägaufnahmen (b, c). Beschwerdefreiheit nach Pars-Infiltration. Rekonstruktion der Pars interarticularis nach Buck (d, e, f) [1].

illeolumbalen Ligaments bzw. des Processus transversus hervorgerufen werden.

\section{Veränderung des sagittalen Profils}

Die Veränderung des sagittalen Profils mit der daraus resultierenden sagittalen oder frontalen Imbalance trägt ebenfalls regelhaft zur Beschwerdesymptomatik bei. Typische klinische Bilder sind hier die Hyperlordosierung der unteren LWS, evtl. mit Sprungschanzenphänomen, sowie die daraus resultierende Dysbalance der spinopelvinen Achse und der unteren Extremität. Auch eine Formabweichung im frontalen Profil mit begleitenden skoliotischen Fehlstellungen ist bei höhergradiger Spondylolisthese häufig.

\section{Diagnostik}

Die Röntgenuntersuchung der Lendenwirbelsäule und des lumbosakralen Übergangs in 2 Ebenen ist integraler Bestandteil der Diagnostik. Hier sollte vor allen Dingen auf lumbosakrale Übergangsstörungen geachtet werden. Von eher historischer Bedeutung ist die Schrägaufnahme der Lendenwirbelsäule, in der der Parsdefekt als „Hundehalsband“ abgebildet werden kann (Abb. 2 b und c). Von großer Bedeutung wiederum sind Wirbelsäulenganzaufnahmen, die das frontale und sagittale Wirbelsäulenprofil darstellen. Anhand dieser Röntgendiagnostik kann eine Vielzahl von lumbalen und lumbopelvinen Parametern bestimmt werden, die in der Diagnostik, aber auch Therapieplanung von Bedeutung sind. Hierzu gehört z. B. die Einteilung der Schweregrade der Spondylolisthese nach Sir Henry William Meyerding [10]. Der Schweregrad der Spondylolisthese wird darüber hinaus auch häufig in Prozentangaben wiedergegeben. Von Bedeutung sind außerdem „slip angle“, „sacral slope“, „pelvic inclination“, „pelvic incidence“, „severity index" sowie die Bestimmung der instabilen Zone (unstable zone) nach Lamartina [7]. Von herausragender Bedeutung für die operative Therapieplanung ist dabei der zuletzt genannte Parameter, da er dabei behilflich ist, das Ausmaß der Instrumentierung, speziell den Entschluss zur Integration des Bewegungssegments L4/ 5 in die Instrumentierung, zu stützen.

Kernspintomografie und Computertomografie sind Standarddiagnostika, die nicht nur Hinweise über das Alter der Spondylolyse, sondern auch über das Defektausmaß liefern. 


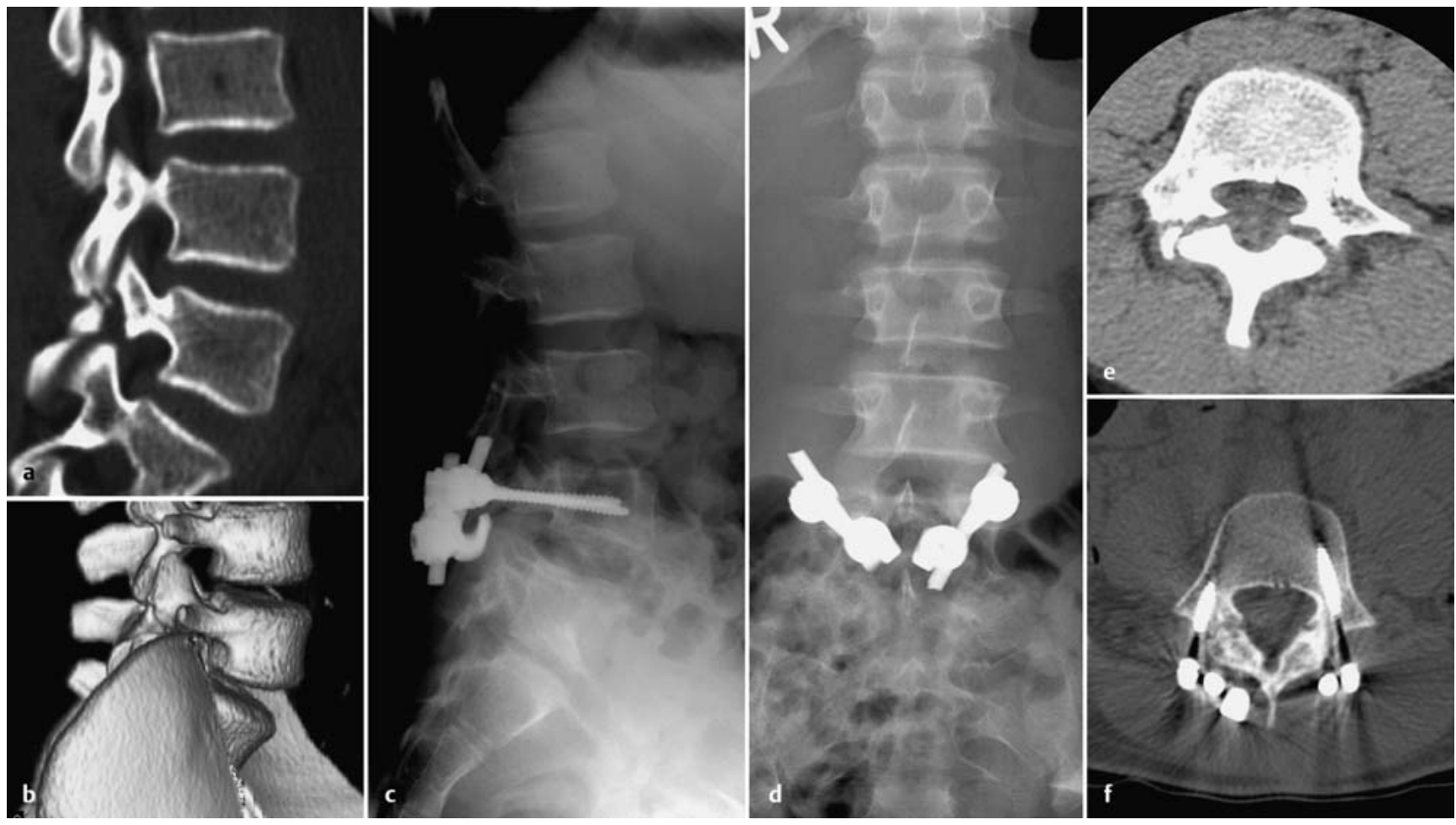

Abb. $\mathbf{3}$ a bis $\mathbf{f}$ 13-jähriger Knabe mit symptomatischer Spondylolyse (a, b). Beschwerdefreiheit nach Pars-Infiltration. Rekonstruktion der Pars interarticularis mit Pedikelschrauben und Laminahacken (c, d). Ausheilung des Pars-Defekts nach 9 Monaten (Vergleich von e und f) [4].

In Zweifelsfällen ist auch eine Szintigrafie oder SPECT (single photon emission computer tomography) sinnvoll, da sie die Bestimmung des Alters der Spondylolyse erlaubt. Dies ist vor allen Dingen für rekonstruktive Eingriffe von Bedeutung.

Als weitere invasive Diagnostika, ggf. auch als Therapeutika, stehen die Infiltration der Pars interarticularis zur lokalen Schmerztherapie, die Diskografie zur Beurteilung des Bandscheibenschadens, die periradikuläre Infiltration der Nervenwurzeln ebenfalls zur Schmerztherapie und die Myelografie, vor allen Dingen bei höchstgradigen Stenosen und Revisionsoperationen, zur Verfügung.

\section{Konservative Therapie}

$80 \%$ aller Patienten mit einer Spondylolyse sind und bleiben asymptomatisch und bedürfen keiner speziellen Therapie.

Symptomatische Patienten, bei denen eine Spondylolyse nebenbefundlich diagnostiziert wird, fallen häufig in die Kategorie des sog. „unspezifischen Rückenschmerzes" und bedürfen keiner speziellen Therapiemaßnahmen. Nur der kleine Prozentsatz symptomatischer Patienten mit „spezifischem Rückenschmerz“ bedarf einer speziellen konservativen The-
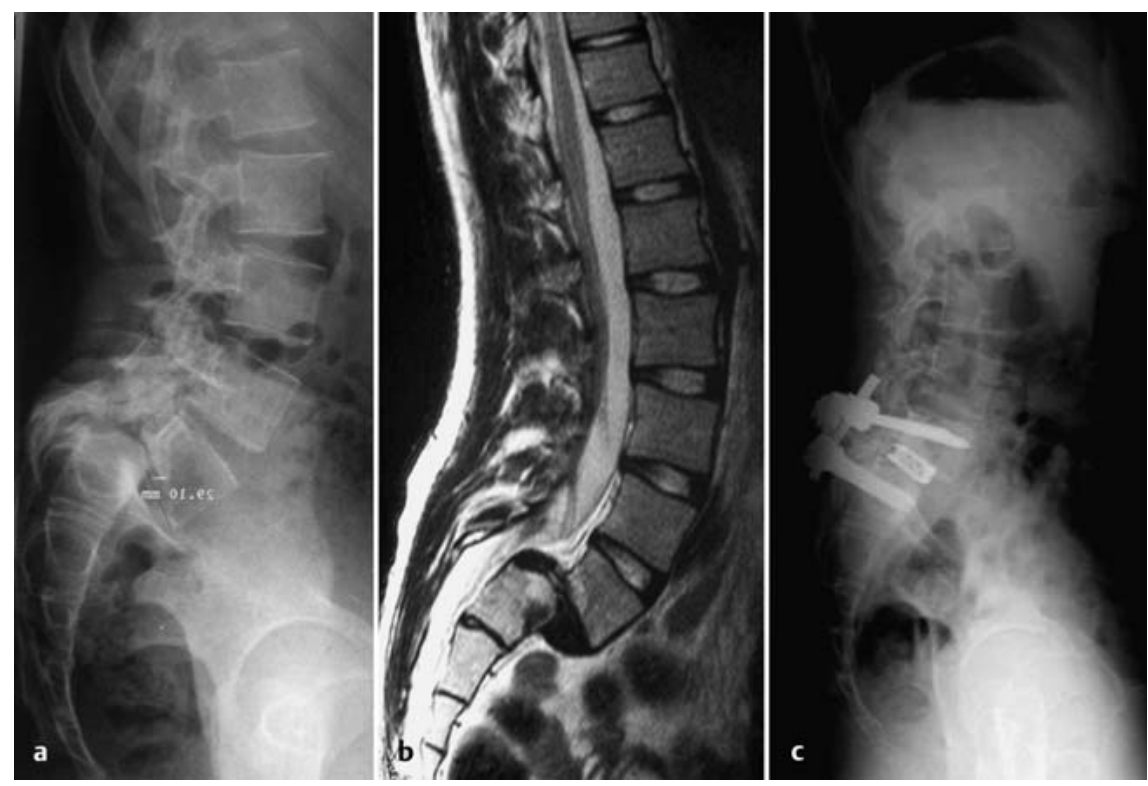

Abb. 4a bis c 17-jähriges Mädchen mit symptomatischer Spondylolyse und Spondylolisthese Meyerding Typ IV (sensibles L5-Syndrom beidseits) (a, b). Postoperatives radiologisches Ausheilungsergebnis nach Fusionsoperation mit Reposition in PLIF-Technik (c).

rapie. Diese besteht in der Applikation von nicht steroidalen Antirheumatika zur Schmerztherapie. Auch im Rahmen der Infiltrationstherapie der Nervenwurzeln bzw. der Parsregion kann eine deutliche Beschwerdelinderung erzielt werden. Speziell im Kindes- und Jugendalter und bei Hinweis auf eine frische Spondy- lolyse kann eine Korsett- und Gipstherapie sinnvoll sein. Generell ist ein Muskelaufbau der Rücken-, aber vor allen Dingen auch Bauchmuskulatur zu empfehlen.

Bei symptomatischen Patienten mit Spondylolyse ist eine radiologische Ver- 

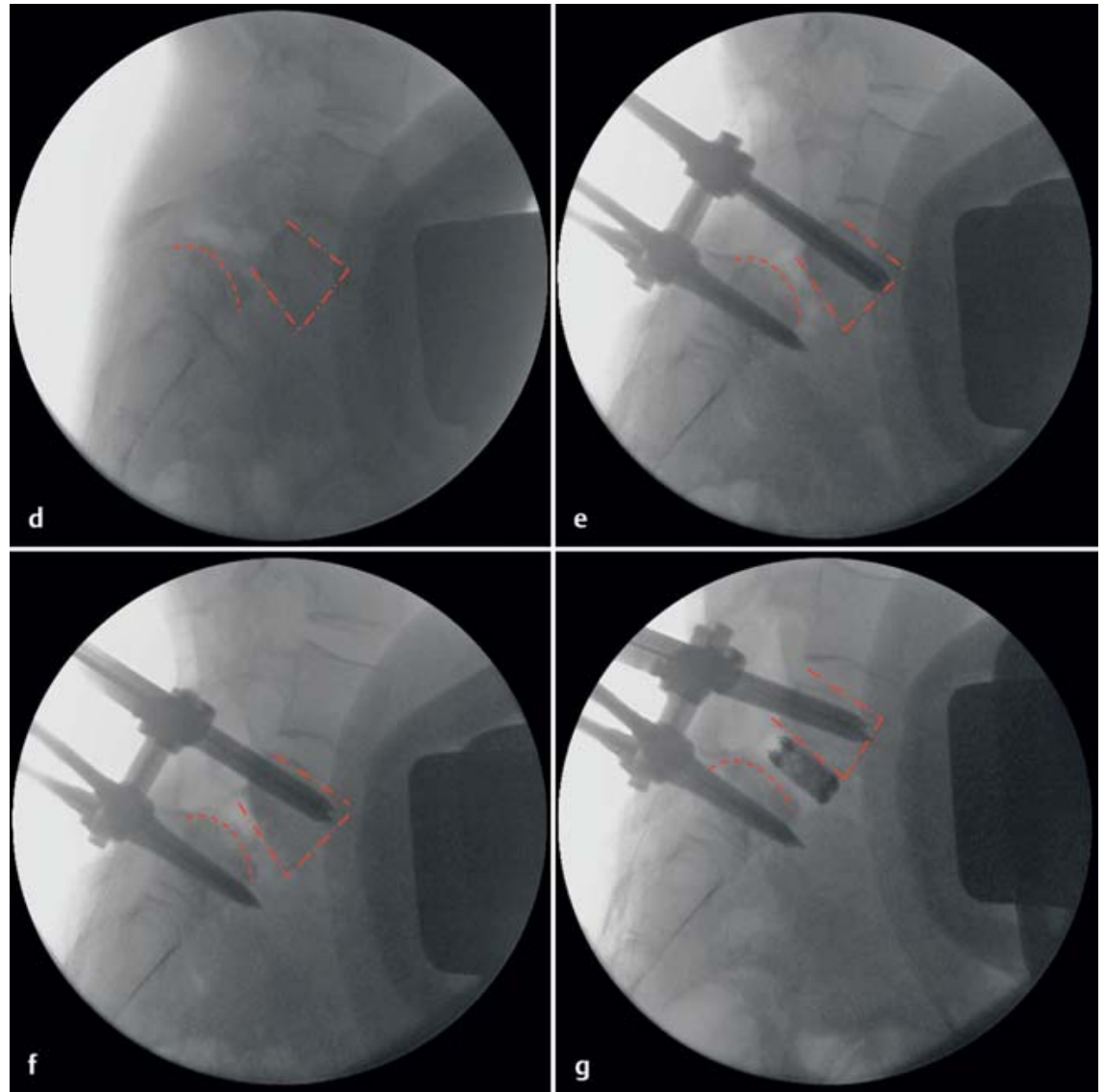

Abb. $\mathbf{4 d}$ bis $\mathbf{g}$ Intraoperative BV-Aufnahme zur Dokumentation der Repositionsschritte.
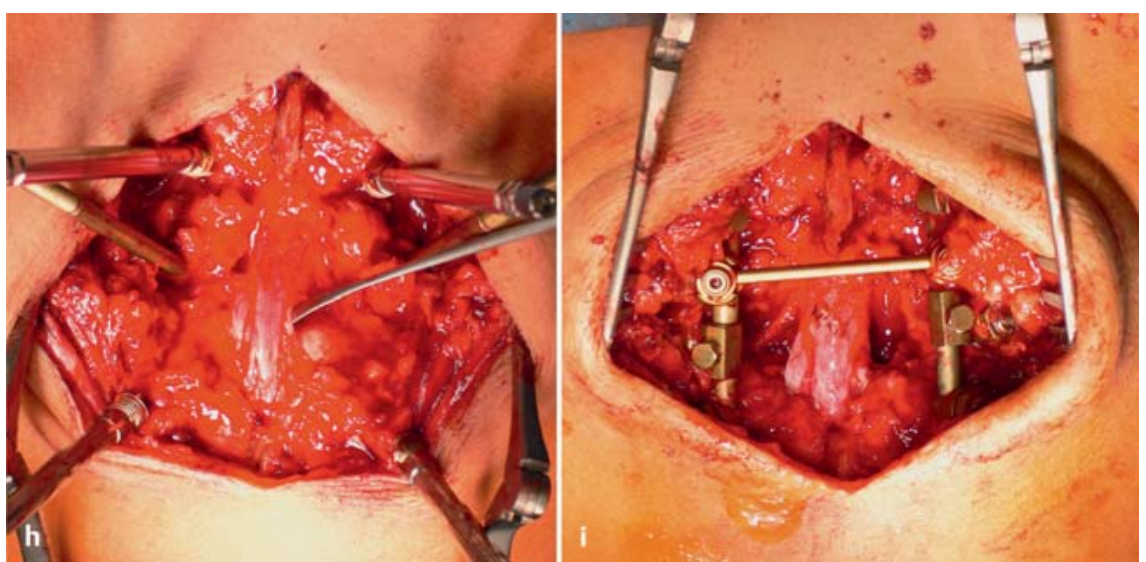

Abb. $\mathbf{4 h}$ und $\mathbf{i}$ Intraoperative klinische Bilder, die den über das Sakrum gespannten Duralsack mit S1-Wurzel vor Reposition (h) und den entspannten Duralsack mit S1-Wurzel nach Reposition (i) zeigen.

laufskontrolle im jährlichen Abstand sinnvoll, um eine Progression der Spondylolisthese auszuschließen. Risikofaktoren für eine Progression sind ein junges Lebensalter, das weibliche Geschlecht, ein dysplastischer Typ (Typ I nach Wiltse), ein domförmiges Sakrum, ein trapezoidförmiger 5. Lendenwirbelkörper, ein Ausmaß der Spondylolisthese vom Typ ausgeprägten Haltungsstörungen sowie beim Auftreten eines neurologischen Defizits.

Prinzipiell können 2 operative Therapieformen, die Rekonstruktion der Pars interarticularis sowie die fusionierenden Operationen, unterschieden werden.

\section{Rekonstruktion der Pars interarticularis}

Ein rekonstruktiver Eingriff im Bereich der Pars interarticularis ist nur dann sinnvoll, wenn es sich um junge Patienten handelt (jünger als 25 Jahre), nur eine minimale Spondylolisthese vorliegt (weniger als $2 \mathrm{~mm}$ Wirbelgleiten), keine wesentlichen Facettendegenerationen und keine wesentlichen Bandscheibendegenerationen des Bewegungssegments existieren.

Der entscheidende Vorteil der Rekonstruktion der Pars interarticularis besteht darin, dass keine Versteifung des Bewegungssegments erforderlich ist.

Zur Rekonstruktion der Pars interarticularis wurden zahlreiche Operationsverfahren beschrieben; allen Verfahren gemeinsam ist, dass das fibrokartilaginäre Gewebe im Bereich der Pars interarticularis ausgeräumt wird, die Knochenenden angefrischt werden, eine Spongiosaplastik im Bereich des Defekts durchgeführt wird und anschließend eine Kompression durch Implantate auf die Knochenenden vorgenommen wird. Die Kompression und Stabilisierung wird entweder durch eine direkte Verschraubung oder Verdrahtung erzielt bzw. mit Spezialimplantaten vorgenommen. Im Einzelnen sind die Verfahren nach Scott [12], Buck [1] (siehe Abb.2), Morscher [11] und Lamartina [7] beschrieben. Das derzeit verbreiteteste Operationsverfahren besteht in einer Rekonstruktion und Stabilisation des Parsdefekts mittels Pedikelschrauben und Laminahaken [4] (Abb. 3).

\section{Fusionsoperationen}

Eine Fusion des lumbosakralen Übergangs kann bei Spondylolyse und Spondylolisthese mit oder ohne Reposition durchgeführt werden. Wenn keine Reposition vorgenommen wird, spricht man von einer In-situ-Spondylodese. Die repositionierenden Verfahren können in unterschiedlichen Techniken, z.B. als ALIF, PLIF (Abb. 4), TLIF (Abb. 5) oder als Gaines-Procedure [2], durchgeführt wer- 


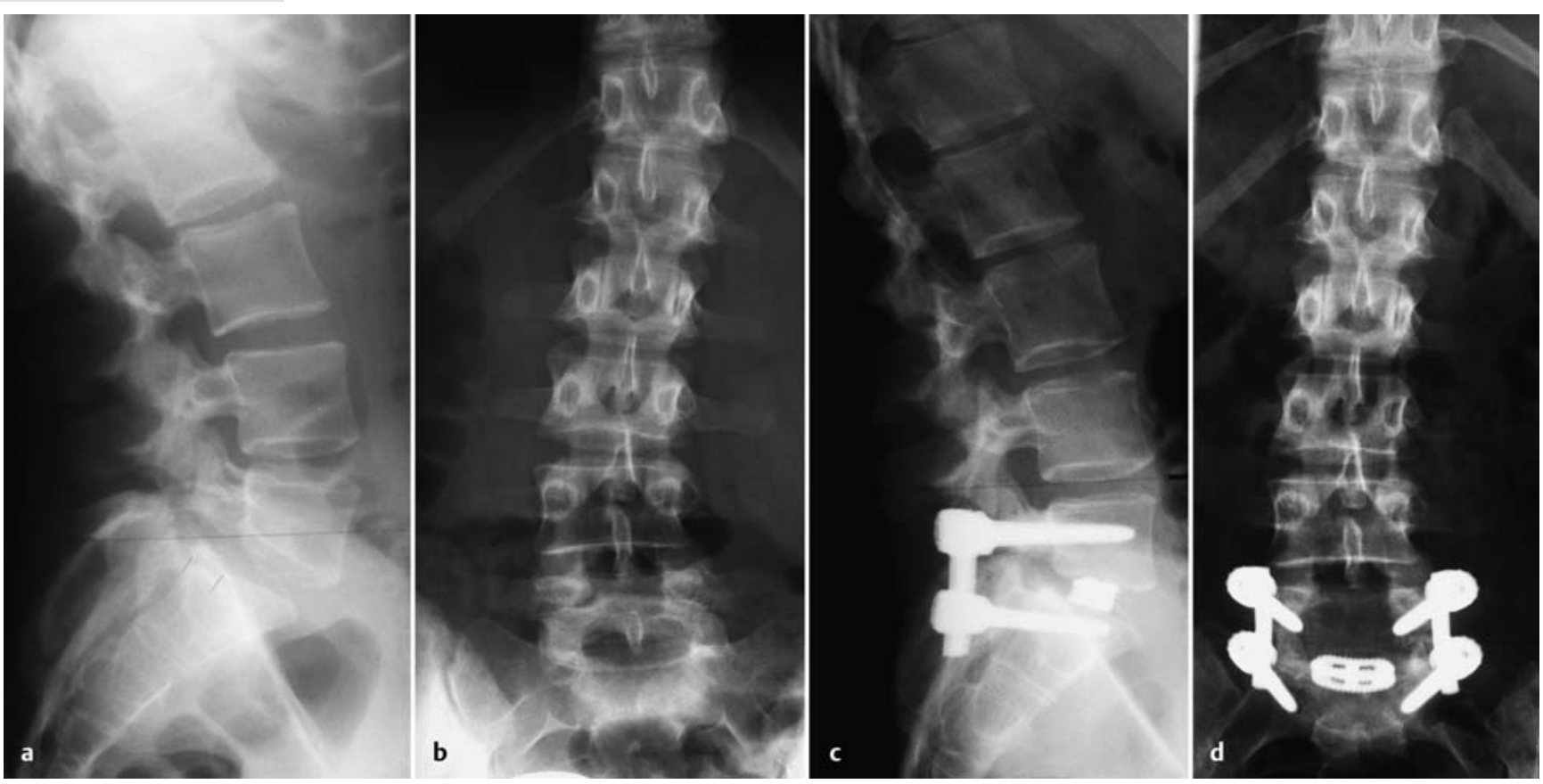

Abb. 5 a bis d 24-jährige Frau mit symptomatischer Spondylolyse und Spondylolisthese Meyerding Typ II (sensibles L5-Syndrom bds. und chronischer belastungsabhängiger Lumbalgie, Skoliose) (a, b). Postoperatives radiologisches Ergebnis nach Fusionsoperation mit Reposition in TLIFTechnik (c, d).

den. Eine generelle Empfehlung hinsichtlich des „optimalen“ Operationsverfahrens existiert derzeit nicht [6].

\section{Ergebnisse der Pars-Rekonstruktion}

Zahlreiche Untersuchungen konnten nachweisen, dass die unterschiedlichen Rekonstruktionsverfahren relativ uniforme Ergebnisse liefern [13]. In 80-90\% der Fälle finden sich gute und sehr gute klinische Ergebnisse. Die Fusionsrate beträgt nahezu unabhängig vom Verfahren ca. $80 \%$. Ein deutlicher Zusammenhang findet sich jedoch zwischen dem Zeitpunkt des Operationsverfahrens und der Fusionsrate; so konnte Hefti [5] nachweisen, dass bei Patienten unter 20 Jahren hohe Fusionsraten zu erzielen sind, wohingegen bei Patienten jenseits des 20. Lebensjahrs Fusionsraten von nur ca. 70\% zu erreichen waren.

\section{Ergebnisse der Fusionsoperationen}

Bei Spondylolyse und Spondylolisthese konnte gezeigt werden, dass die Fusionsoperation effektiver ist und bessere Ergebnisse zeigt als die konservative Therapie [6].

Die Fusionsergebnisse sind dabei aber entscheidend vom Ausmaß der Spondylolisthese abhängig. Sog. geringgradige Spondylolisthesen (Meyerding Grad I und II, Abb.5) zeigen Fusionsraten von
80-95\% bei guten und exzellenten Ergebnissen, die auch im Bereich von 8090\% liegen. Eine gute Zusammenfassung der klinischen Ergebnisse in Bezug auf die unterschiedlichen Operationsverfahren findet sich bei Jakobs [6].

Bei höhergradigen Spondylolisthesen (Meyerding Grad III und IV) finden sich ähnliche klinische Gesamtergebnisse wie bei geringgradigen Spondylolisthesen [3], aber höhere Komplikationsraten (s.u.). Unklar ist nach wie vor der Stellenwert der Reposition bei den hochgradigen Spondylolisthesen (Meyerding Grad III und IV, Abb.4). Obwohl viele theoretisch gute Argumente für eine Reposition sprechen (Wiederherstellung des Wirbelsäulenprofils, Dekompression der Nerven, Rekonstruktion der spinopelvinen Achse etc.), konnte in den bisher vorliegenden Fallkontrollstudien kein signifikanter Unterschied hinsichtlich des klinischen Outcomes beim Vergleich der Methoden nachgewiesen werden [15]. Auffällig ist aber eine geringere Pseudarthroserate bei Reposition und instrumentierter Fusion.

\section{Komplikationen}

Vor allen Dingen bei der Reposition höhergradiger Spondylolisthesen handelt es sich um sehr aufwendige Operationsverfahren, die eine Vielzahl von Komplikationsmöglichkeiten bieten. Typische
Komplikationen sind die Pseudarthrose sowie die Anschlussproblematik im Sinne eines „adjacent segment syndrome“ bzw. eines „transition zone syndrome“. Eine typische und häufige Komplikation ist ein neurologisches Defizit, das in der Regel die L5-Nervenwurzel betrifft. Typisch ist hier vor allen Dingen auch das L5-Defizit, das durch Release des ileolumbalen Ligaments von ventral behoben werden kann [14]. Eine weitere typische Komplikation speziell der hochgradigen Spondylolysen und Spondylolisthesen ist die sog. S1-/S2-Deformität.

\section{Fazit}

Die Spondylolyse und Spondylolisthese ist ein komplexes Krankheitsbild, das häufig bereits im Kindes- und Jugendalter relevant wird. Nur die symptomatischen Spondylolisthesen bedürfen der Therapie. Speziell bei rascher Progression der Spondylolisthese oder dem Vorliegen von Risikofaktoren ist eine Behandlung in einem spezialisierten Zentrum sinnvoll. Sowohl die rekonstruktiven Eingriffe im Bereich der Pars interarticularis als auch die Fusionsoperation bei hochgradigen Spondylolisthesen sind schwierig und komplikationsträchtig. Bei rechtzeitiger und adäquater Behandlung lassen sich jedoch regelhaft gute und sehr gute Ergebnisse erzielen. 


\section{Literatur}

${ }^{1}$ Buck JE. Direct repair of the defect in spondylolisthesis. J Bone Joint Surg [Br] 1970; 52: 432-437

2 Gaines RW. The L5 vertebrectomy approach for the treatment of spondyloptosis. In: Bridwell KH, DeWald RL, eds. The textbook of spinal surgery. 2nd ed. Philadelphia: LippincottRaven; 1997: 1337-1348

${ }^{3}$ Gaines RW. L5 vertebrectomy for the surgical treatment of spondyloptosis: thirty cases in 25 years. Spine 2005; 30: S66-S70

${ }^{4}$ Gilet P, Petit M. Direct repair of spondylolisis without spondylolisthesis, using a rod-screw construct and bone grafting of the pars defect. Spine 1999; 24: 1252-1256

${ }^{5}$ Hefti F, Seelig W, Morscher E. Repair of lumbar spondylolisis with hook-screw. Int Orthop 1992; 16: 81-85

${ }^{6}$ Jacobs WCH, Vreeling A, De Kleuver M. Fusion for low-grade isthmic spondylolisthesis: a systematic review of the literature. Eur Spine J 2006; 15: 391-402

7 Lamartina C. A square to indicate the unstable zone in severe spondylolisthesis. Eur Spine J 2001; 10: 444-448

8 Logroscino G, Mazza O, Aulisa G et al. Spondylolysis and spondylolisthesis in the paediatric and adolescent population. Childs Nerv Syst 2001; 17: 644-655

9 Marchetti PG, Bartolozzi P, Binazzi R et al. Preoperative reduction of spondylolisthesis. Chir Organi Mov 2002; 87: 203-215

${ }^{10}$ Meyerding HW. Spondylolisthesis. Surg Gynecol Obstet 1932; 54: 371-377

${ }^{11}$ Morscher E, Gerber B, Fasel J. Surgical treatment of spondylolisthesis by grafting and direct stabilisation spondylolysis by means of a hook screw. Arch Orthop Trauma Surg 1984; 103: $175-178$

${ }^{12}$ Nicol RO, Scott JH. Lytic spondylolisthesis. Repair by wiring. Spine 1986; 10: 185-188

13 Saraste $H$. Long-term clinical and radiological follow-up of spondylolysis and spondylolisthesis. J Paediatr Orthop 1987; 7: 631-638

${ }^{14}$ Sasso RC, Kozak JA, Dickson JH. The sickle ligament revisited. Release of the lumbosacral ligament via an anterior approach. Spine 1993; 18: 2127-2130

15 Transfeldt EE, Mehbod AA. Evidence based medicine analysis of isthmic spondylolisthesis treatment including reduction versus fusion in situ for high-grade slips. Spine 2007; 32: S126-S129

${ }^{16}$ Wiltse LL, Rothman LG. Spondylolisthesis: classification, diagnosis, and natural history. Seminars in Spine Surgery 1968; 1: 78-94
Priv.-Doz. Dr. med. Frank Kandziora

Chefarzt

Dr. med. Matti Scholz

Facharzt

Dr. med. Andreas Pingel

Oberarzt

Interdisziplinäres Zentrum

für Wirbelsäulenchirurgie und

Neurotraumatologie

Prof. Dr. med. Reinhard Hoffmann

Ärztlicher Direktor

Chefarzt der Abteilung für Unfallchirurgie und Orthopädische Chirurgie

BG-Unfallklinik

Friedberger Landstraße 430

60389 Frankfurt am Main

ws@bgu-frankfurt.de 